\title{
The symbiotic relationship of suspended sediment and pathogenicity in freshwater ecosystems
}

\author{
DANIELLE VANMENSEL ${ }^{1}$, IAN DROPPO ${ }^{2}$ AND \\ CHRISTOPHER WEISENER ${ }^{1}$ \\ ${ }^{2}$ Environment and Climate Change Canada \\ Presenting Author: vanmensd@uwindsor.ca
}

${ }^{1}$ University of Windsor

The introduction and proliferation of pathogenic organisms in aquatic systems is a serious global issue that consequently leads to several economic, financial, and health concerns. Health and safety related to recreational water use can be monitored through water quality assessments but are typically simple culture-based identification tests of fecal indicator bacteria, which are outdated and misleading. These traditional methods merely focus on broad taxa groups and both the suspended sediment (SS) and bed sediment compartments are often overlooked. Furthermore, they provide no insight into the active microbial community or the source of contamination. To help bridge this knowledge gap, our study aimed to 1) examine the metatranscriptome of the microbial community associated with SS in freshwater lotic systems; and 2) explore the influence of the transported SS metatranscriptome in tributaries adjacent to nearshore freshwater beaches. Samples were collected seasonally from Lake St. Clair and Lake Erie (part of the Laurentian Great Lakes in Southern Ontario, Canada), allowing for spatial and temporal comparisons. The beaches in this region are influenced by both agriculture runoff and continued urban expansion. Results show that both the adjacent tributary and beach SS have similar microbial functional diversity with strong correlations by site. We identified expression of transcripts related to nine bacterial infectious disease pathways in all of our samples, with legionellosis $(s d h A)$ and Vibrio cholera pathogenesis at the forefront. Lake samples typically showed higher overall expression $(\mathrm{p}<0.05)$ of infectious disease pathways compared to tributary, and summer samples displayed upregulation $(\mathrm{p}<0.05)$ compared to fall. Our data suggests that SS transport acts as a strong vector for pathogen transport as well, making this facet an important area for further research as it pertains to human health with regards to recreational water use. To our knowledge, this work is the first to investigate SS as a source of bacterial pathogenicity in aquatic microbial communities using metatranscriptomic analyses and has significant implications to help address growing issues of microbial contamination impacting freshwater security. 